\title{
Provisorische Regelung der Anerkennung von Notfallkursen
}

\author{
Arbeitsgruppe Weiterbildung der SGAM
}

Da per 1. Januar 2000 die Übergangsfrist abgelaufen ist, muss für die Erlangung des Facharzttitels FMH für Allgemeinmedizin der Nachweis erbracht werden, dass ein von der SGAM organisierter oder anerkannter Kurs in Notfallmedizin absolviert wurde.

Von der FMH aus sind Bestrebungen im Gang, die Anerkennung/Zertifizierung generell zu regeln, es ist aber noch nicht abzusehen, wann diese Organisation funktionsfähig sein wird. Die SGAM mit ihrer Arbeitsgruppe Weiterbildung hat deshalb folgende provisorische Regelung der Anerkennung der Ausbildung in Notfallmedizin getroffen:

1. Die Gesuche um Anerkennung sind unter Beilage des Kursprogramms vor der Absolvierung an das Sekretariat der SGAM (L. Schneider, Oberplattenstrasse 73, 9620 Lichtensteig, Fax 07198866 41, E-mail sgam@hin.ch) oder an Dr. med. A. Früh, Talweg 6, 4437 Waldenburg (Fax 06196184 95, E-mail acfrueh@swissonline.ch) zu richten. Ausnahmen siehe Punkt 3.

2. Wegleitend für die Anerkennung sind die unten stehenden "Anforderungen an Notfallmedizinkurse"

3. Die Ausbildung der MEDIFAN sowie der vorgesehene ANAK-Kurs der Schweizerischen Armee gelten als provisorisch anerkannt, dort genügt es, die Bestätigung der (vollständigen!) Absolvierung an das Sekretariat der SGAM zu senden.

Korrespondenz:

Dr. med. A. Früh

Talweg 6

CH-4437 Waldenburg

\section{Anforderungen an Notfallmedizinkurse}

Inhalt und Zeitbedarf

Folgende Notfallsituationen müssen beherrscht werden können:

- Bewusstlosigkeit,

- respiratorische Störungen,

- zirkulatorische Störungen,

- kardiale Störungen,

- Kreislaufstillstand,

- Polytrauma,

- Schädelhirntrauma.

Dafür müssen folgende Kompetenzen vermittelt werden (in Klammern Richtwert für den Zeitaufwand der einzelnen Punkte):

- Beurteilung der Vitalfunktionen (1 Std.),

- Bergung/Lagerung/Polytrauma (3 Std.),

- Airway-Management/Beatmung (4 Std.),

- Schockbehandlung/IV-Leitungen (2 Std.),

- Rhythmusstörungen (2 Std.),

- Reanimation: BLS + Defibrillation (4 Std.),

- Analgesie (1 Std.),

- Notfallmedikamente (1 Std.),

- Equipment für den Notfallarzt (2 Std.),

- entsprechende Massnahmen für den pädiatrischen Notfall (4 Std.),

- Megacode-Training (6 Std.).

Minimale Gesamtdauer somit: 30 Std. Die Kurse können modular aufgebaut sein, wobei ein Modul eine Dauer von 6 Stunden nicht unterschreiten soll.

\section{Methodik}

Da es sich beim Kursinhalt überwiegend um Fertigkeiten und Abläufe handelt, die im Ernstfall automatisch richtig ausgeführt werden müssen, sollen mindestens 75\% der Zeit für die praktische Arbeit in der Gruppe aufgewendet werden. Eine Gruppe soll maximal aus 6 Teilnehmern bestehen, das Instruktorenzu-Teilnehmer-Verhältnis somit 1:6. Eine praktische Schlussprüfung zur Selbsteinschätzung wird empfohlen. 


\section{Facharztprüfung zur Erlangung des Facharzttitels Physikalische Medizin und Rehabilitation und des Europäischen Facharzttitels für Physikalische Medizin und Rehabilitation}

Aufgrund des Weiterbildungsprogrammes, welches am 1. Januar 2000 in Kraft gesetzt wurde, ist die Teilnahme an der Facharztprüfung Voraussetzung für die Erteilung des Facharzttitels Physikalische Medizin und Rehabilitation. Es empfiehlt sich, die Facharztprüfung frühestens im letzten Jahr der reglementarischen Weiterbildung abzulegen (Art. 23 WB0).
Spezialärztinnen und Spezialärzte für Physikalische Medizin und Rehabilitation sowie Kandidatinnen und Kandidaten haben gleichzeitig die Möglichkeit, den Europäischen Facharzttitel PMR zu erlangen.

\section{Schriftliche Prüfung}

Ort: Inselspital, Rheumaklinik, Bern

Datum: Samstag, 25. November 2000

Prüfungsgebühr: Die Schweizerische Gesellschaft für Physikalische Medizin und Rehabilitation erhebt für die schriftliche Prüfung eine Prüfungsgebühr von Fr. 500.-.

Anmeldung: Sekretariat SGPMR, c/o Schweizerische Rheumaliga, Renggerstrasse 71, 8038 Zürich, Tel. 01 48740 00/07, Fax 0148740 19, E-mail srl@srl.ch

Anmeldefrist: 30. September 2000
Examen de spécialiste en vue de l'obtention du titre de spécialiste FMH en médecine physique et réadaptation et du titre européen de médecine physique et réadaptation

Conformément au programme de formation postgraduée entré en vigueur le $1^{\mathrm{er}}$ janvier 2000, la participation à l'examen de spécialiste est une condition requise pour l'obtention du titre de spécialiste FMH en médecine physique et réadaptation. Il est recommandé de passer l'examen de spécialiste au plus tôt durant la dernière année de formation postgraduée réglementaire (art. 23 RFP).
Les spécialistes FMH en médecine physique et réadaptation et les candidats ont la possibilité de passer l'examen européen du titre européen de médecine physique et réadaptation.

\section{Examen écrit}

Lieu: Hôpital de l'Ile, Service de Rhumatologie, Berne Date: Samedi le 25 novembre 2000

Taxe d'examen écrit: La société suisse de médecine physique et réadaptation prélève une participation aux frais de Fr. 500.- pour l'examen écrit.

Inscription: Secrétariat SSMPR c/o Ligue Suisse contre le rhumatisme, Renggerstrasse 71, 8038 Zurich, tél. 0148740 00/07, fax 0148740 19, e-mail: srl@srl.ch

Délais d'inscription: le 30 septembre 2000 